\title{
Analysis of Dual Rotating Rake Data from the NASA Glenn Advanced Noise Control Fan Duct with Artificial Sources
}

\author{
Milo D. Dahl* and Daniel L. Sutliff ${ }^{\dagger}$ \\ NASA Glenn Research Center, Cleveland, OH, 44135, USA
}

\begin{abstract}
The Rotating Rake mode measurement system was designed to measure acoustic duct modes generated by a fan stage. Initially, the mode amplitudes and phases were quantified from a single rake measurement at one axial location. To directly measure the modes propagating in both directions within a duct, a second rake was mounted to the rotating system with an offset in both the axial and the azimuthal directions. The rotating rake data analysis technique was then extended to include the data measured by the second rake. The analysis resulted in a set of circumferential mode levels at each of the two rake microphone locations. Radial basis functions were then least-squares fit to this data to obtain the radial mode amplitudes for the modes propagating in both directions within the duct. Validation experiments have been conducted using artificial acoustic sources. Results are shown for the measurement of the standing waves in the duct from sound generated by one and two acoustic sources that are separated into the component modes propagating in both directions within the duct. Measured reflection coefficients from the open end of the duct are compared to analytical predictions.
\end{abstract}

\section{Introduction}

A rotating rake microphone system developed by the NASA Glenn Research Center has been an aid to advancing the understanding of acoustic modes generated in turbofan engine ducts. ${ }^{1}$ Turbofan noise, in part caused by rotor-stator and other interactions, may couple to rotating duct modes that propagate through and radiate outward from the duct to the far field. The rotating rake system is used to measure and identify these rotating duct modes. Various applications of the rotating rake system are described in Sutliff ${ }^{1}$ including model-scale ducted fans, full-scale turbofan engines, and the NASA Glenn Advanced Noise Control Fan (ANCF) facility.

The ANCF enables the development of fan noise reduction concepts and computational aeroacoustic codes through data provided by unique measurement tools, such as the rotating rake system, giving improved physical understanding and code validation data. Much of this was based on the results measured by one rotating rake. Recently, Dahl et al. ${ }^{2}$ extended the analysis for rotating rake measured data to include a corotating second rake that allows for the separation of modes propagating in both directions within the duct. This paper provides an analysis of measured data take by Sutliff ${ }^{3}$ in the ANCF with one and two rotating rakes to help validate the measurement methodology and data processing. The experiments used two sets of artificial acoustic sources to generate propagating rotating modes. Results of data analysis are shown for the standing wave generated in the duct when one acoustic source is operating and the propagating modes reflect back into the duct from the open end. Reflection coefficients were determined and those results are compared to analytical predictions for open end duct reflection coefficients. Finally, results for the two-rake system measuring the acoustic field between two artificial sources are given where one source varies in amplitude.

\footnotetext{
* Senior Research Scientist, Acoustics Branch, Senior Member AIAA

$\dagger$ Aerospace Engineer, Acoustics Branch, Associate Fellow AIAA
} 


\section{Rotating Rake Data Analysis}

The goal of analyzing rotating rake data is to obtain the amplitude for each of the measured modes. When data from one rotating rake is analyzed, the modal amplitudes are a result of the superposition of modes propagating in both directions within the duct. The amplitudes of the measured modes that propagate in both directions in the duct are obtained separately when data from two rotating rakes are analyzed. Once the mode amplitudes are found, the acoustic power in each mode can be computed. For that to occur, two basic processes are followed: 1) determine the radial basis functions by numerically solving the eigenvalue problem presented by the governing equation and 2) determine the mode amplitudes by a least-squares fit using a system of equations representing modes propagating in one or both directions in the duct for data collected from one or two rotating rakes, respectively. The presence of decaying or evanescent modes are also accommodated in the fit for the case using the data from two rotating rakes.

The numerical analysis of the eigenvalue problem is based on a discretization of the third order equation for the acoustic pressure derived from combining the linearized momentum and energy equations. The flow is compressible and the mean flow variables are a function of the radius. Using separation of variables, the partial differential equation is reduced to an ordinary second order differential equation for the radial basis function. Along with the appropriate equations for the boundary conditions, discretization is applied resulting in a matrix equation that is solved for a set of complex eigenvalues and their associated radial basis functions. The complex eigenvalue is identified as the axial wave number $k_{m n}$ that determines the axial variation with distance of the acoustic pressure and whether the particular acoustic mode with circumferential mode number $m$ and radial mode number $n$ propagates and/or decays with axial distance. The associated basis function $E_{m n}(r)$ for each $k_{m n}$ is only a function of the duct radius. The details for a finite difference approach are given in Dahl \& Sutliff ${ }^{4}$ and in Dahl et al. ${ }^{2}$ For a constant mean flow, Sutliff ${ }^{5}$ gives the exact, closed-form solution.

\section{II.A. Single Rake Analysis}

The measured rotating rake microphone data are analyzed to obtain the total circumferential mode amplitude $C_{m}^{s}\left(r_{i}\right)$ at each radial measurement location $r_{i}$ prior to further decomposition using the radial basis functions. Consider the signal

$$
p(x, \theta, r, t)=\Re\left\{\sum_{s} \sum_{m=-M}^{M} C_{m}^{s}(x, r) e^{-j 2 \pi s B \Omega t} e^{j m \theta}\right\}
$$

where the frequency $s B \Omega$ is related to a rotating fan with $\Omega$, the shaft speed in revolutions per second, $B$, the number of blades, and, $s$, the harmonic number $s=1,2, \ldots$. The signal is sampled synchronously with the shaft speed and ensemble averaged over a period $T=K \Delta t$. This reduces the uncorrelated broadband noise in favor of the tonal content of the signal. The circumferential mode index $m$ ranges over a finite number of modes denoted by the general value $M$.

Each microphone in a rake is generating a signal that is described by equation (1). The rake is located at

$$
\begin{aligned}
& x=x_{1}=\text { axial location } \\
& \theta=\theta_{1}+\psi \text { offset azimuthally from some reference }
\end{aligned}
$$

and the microphones are distributed along the rake

$$
r=r_{i}=\text { microphones at different radii } r_{i}, i=1,2, \ldots, N_{1}
$$

The rake rotates according to $\theta_{1}=2 \pi \Gamma t$ where $\Gamma$ is the rake rotational speed. Equation (1) becomes

$$
\begin{aligned}
p\left(x_{1}, \theta, r_{i}, t\right) & =\Re\left\{\sum_{s} \sum_{m=-M}^{M} C_{m}^{s}\left(x_{1}, r_{i}\right) e^{-j 2 \pi s B \Omega t} e^{j m \theta}\right\} \\
& =\Re\left\{\sum_{s} \sum_{m=-M}^{M} C_{m}^{s}\left(x_{1}, r_{i}\right) e^{-j 2 \pi s B \Omega t} e^{j m\left(\theta_{1}+\psi\right)}\right\} \\
& =\Re\left\{\sum_{s} \sum_{m=-M}^{M} C_{m}^{s}\left(x_{1}, r_{i}\right) e^{j m \psi} e^{-j 2 \pi(s B \Omega-m \Gamma) t}\right\}
\end{aligned}
$$


Taking the discrete Fourier transform for all the rake microphone signals represented by equation (2), we obtain for any spectral component of the signals:

$$
\begin{aligned}
\hat{P}_{m}^{s}\left(x_{1}, \psi, r_{i}, \ell\right) & =\frac{1}{2} C_{m}^{s}\left(x_{1}, r_{i}\right) e^{j m \psi} h(n-\ell)+\frac{1}{2} C_{m}^{s *}\left(x_{1}, r_{i}\right) e^{-j m \psi} h(K-n-\ell) \\
\ell & =0,1,2, \ldots, K-1
\end{aligned}
$$

where $h$ is a function representing spectral leakage if the tones are not centered in the frequency bins. That is, if

$$
\ell=n=n_{r}\left(\frac{s B \Omega}{\Gamma}-m\right)
$$

where $n_{r}$ is the number of rake revolutions in the time history $T$ and the right side is an integer, then $h \rightarrow 1$ and there is no spectral leakage. Equation (3) shows that the spectrum contains families of spikes about each harmonic $s$ having the amplitudes of the $\pm m$ circumferential modes. These amplitudes can be simply extracted using

$$
C_{m}^{s}\left(x_{1}, r_{i}\right)=2 \hat{P}_{m}^{s}\left(x_{1}, \psi, r_{i}, \ell\right) e^{-j m \psi}
$$

In current practice, the signals are processed such that $C_{m}^{s}=2 \hat{P}_{m}^{s}$ and the rotation by $e^{-j m \psi}$ is applied during the solution for the radial modes as shown in equation (7).

For a single rake, the Fourier analysis of a rotating rake microphone signal has provided the complex total circumferential mode amplitude $C_{m}^{s}\left(r_{i}\right)$ at each radial location, $r_{i}$. These values are expected to be functions of the radial mode content in the duct following

$$
C_{m}^{s}\left(x_{1}, r_{i}\right)=\sum_{n=0}^{N_{\max }-1} P_{s m n} E_{s m n}\left(r_{i} ; x_{1}\right) \quad i=1,2, \ldots, N_{1}
$$

Equation (5) represents the attempt to further decompose the measured circumferential mode data into an approximate series of weighted radial basis functions $E_{s m n}\left(r_{i} ; x_{1}\right)$ computed using the numerical method to solve the eigenvalue problem. The radial basis function was computed for a frequency with harmonic number $s$ for the duct condition at the rake axial location $x_{1}$. To solve equation (5) for the complex radial mode amplitudes, we use the least-squares method. The solution is to minimize the residual sum of squares

$$
e=\|\mathbf{c}-\mathbf{B P}\|_{2}^{2}
$$

where

$$
\mathbf{c}=\left[\begin{array}{lll}
C_{m}^{s}\left(x_{1}, r_{1}\right) & \ldots & C_{m}^{s}\left(x_{1}, r_{N_{1}}\right)
\end{array}\right]^{\mathrm{T}}
$$

is a vector comprised of the measured complex circumferential mode data of length $n_{p}=N_{1}$,

$$
\mathbf{B}=\left[\begin{array}{ccc}
E_{s m 0}\left(r_{1} ; x_{1}\right) & \ldots & E_{s m\left(N_{\max }-1\right)}\left(r_{1} ; x_{1}\right) \\
\vdots & \ldots & \vdots \\
E_{s m 0}\left(r_{N_{1}} ; x_{1}\right) & \ldots & E_{s m\left(N_{\max }-1\right)}\left(r_{N_{1}} ; x_{1}\right)
\end{array}\right]
$$

is a matrix of complex coefficients from the radial basis functions of size $n_{p} \times n_{c}, n_{c}=N_{\max }$, and

$$
\mathbf{P}=\left[\begin{array}{lll}
P_{s m 0} & \cdots & P_{s m\left(N_{\max }-1\right)}
\end{array}\right]^{\mathrm{T}}
$$

is the complex solution vector for the radial mode amplitudes of length $n_{c}$. By ensuring that the number of measurement points $N_{1}$ is greater than the number of radial basis functions $N_{\max }$, the problem is overdetermined, $n_{p}>n_{c}$, and a solution to this linear problem is easily computed using the singular value decomposition method. This method provides the best approximation to solving the linear least-squares problem for over-determined systems and it is robust, handling problems where $\mathbf{B}$ is close to being singular. (See Lawson \& Hanson $^{6}$ for details.) 


\section{II.B. Dual Rake Analysis}

It has been assumed in solving equation (5) for the mode amplitudes $P_{s m n}$, using $N_{\max }$ radial mode shapes, that the mode propagates in only one direction. This is not, in general, the case. Modes may propagate in both the $+x$ direction and the $-x$ direction. To measure these modes, a second rake is required, offset axially and co-rotating with the first rake. The signals from the second rake microphones are acquired simultaneously with the microphone signals from the first rake and Fourier analyzed in the same manner to obtain the total complex circumferential mode amplitudes. The signals at each rake location are the sums of $+x$ direction and $-x$ direction propagating and perhaps evanescent modes. To extract the radial mode amplitudes, the measured circumferential mode amplitudes are written as the sum of radial basis functions as follows:

$$
\begin{aligned}
C_{m}^{s}\left(x_{1}, r_{i}\right)= & \sum_{n=0}^{N_{\max }-1}\left[\left\{P_{s m n}^{+} e^{j k_{1}^{+} x_{1}}\right\} E_{s m n}^{+}\left(r_{i} ; x_{1}\right)+\left\{P_{s m n}^{-} e^{j k_{2}^{-} x_{2}}\right\} E_{s m n}^{-}\left(r_{i} ; x_{1}\right) e^{j\left(k_{1}^{-} x_{1}-k_{2}^{-} x_{2}\right)}\right] \\
C_{m}^{s}\left(x_{2}, r_{i^{\prime}}^{\prime}\right) e^{-j m \psi}= & \sum_{n=0}^{N_{\max }-1}\left[\left\{P_{s m n}^{+} e^{j k_{1}^{+} x_{1}}\right\} E_{s m n}^{+}\left(r_{i^{\prime}}^{\prime} ; x_{2}\right) e^{j\left(k_{2}^{+} x_{2}-k_{1}^{+} x_{1}\right)}+\left\{P_{s m n}^{-} e^{j k_{2}^{-} x_{2}}\right\} E_{s m n}^{-}\left(r_{i^{\prime}}^{\prime} ; x_{2}\right)\right] \\
& i=1,2, \ldots, N_{1} \quad i^{\prime}=1,2, \ldots, N_{2}
\end{aligned}
$$

where $\psi$ is the azimuthal offset between the rake at $x_{1}$ and the rake at $x_{2}$. The axial wave numbers are functions of the mode numbers $m$ and $n$. The sign superscript on $k$ indicates the direction of propagation or decay and the subscripts ' 1 ' and ' 2 ' apply for the solution of the eigenvalue problem at the rake locations $x_{1}$ and $x_{2}$. Also, $r_{i}$ and $r_{i^{\prime}}^{\prime}$ denote that radial locations of microphones on the two rakes may differ in location and number. This formulation rescales the amplitudes and radial basis functions to properly handle evanescent modes and allows for small changes in flow and duct area with axial distance. ${ }^{7}$

To solve equation (7) for the complex radial mode amplitudes, we again use the least-squares method. The solution is to minimize the residual sum of squares, equation (6), where in the dual rake analysis

$$
\mathbf{c}=\left[\begin{array}{llllll}
C_{m}^{s}\left(x_{1}, r_{1}\right) & \ldots & C_{m}^{s}\left(x_{1}, r_{N_{1}}\right) & C_{m}^{s}\left(x_{2}, r_{1}^{\prime}\right) e^{-j m \psi} & \ldots & C_{m}^{s}\left(x_{2}, r_{N_{2}}^{\prime}\right) e^{-j m \psi}
\end{array}\right]^{\mathrm{T}}
$$

is a vector of length $n_{p}=N_{1}+N_{2}$ comprised of the measured complex circumferential mode data,

$$
\mathbf{B}=\left[\begin{array}{ccc}
E_{s m 0}^{+}\left(r_{1} ; x_{1}\right) & E_{s m 0}^{-}\left(r_{1} ; x_{1}\right) e^{j\left(k_{1}^{-} x_{1}-k_{2}^{-} x_{2}\right)} & \ldots \\
\vdots & \vdots & \ldots \\
E_{s m 0}^{+}\left(r_{N_{1}} ; x_{1}\right) & E_{s m 0}^{-}\left(r_{N_{1}} ; x_{1}\right) e^{j\left(k_{1}^{-} x_{1}-k_{2}^{-} x_{2}\right)} & \ldots \\
E_{s m 0}^{+}\left(r_{1}^{\prime} ; x_{2}\right) e^{j\left(k_{2}^{+} x_{2}-k_{1}^{+} x_{1}\right)} & E_{s m 0}^{-}\left(r_{1}^{\prime} ; x_{2}\right) & \ldots \\
\vdots & \vdots & \ldots \\
E_{s m 0}^{+}\left(r_{N_{2}}^{\prime} ; x_{2}\right) e^{j\left(k_{2}^{+} x_{2}-k_{1}^{+} x_{1}\right)} & E_{s m 0}^{-}\left(r_{N_{2}}^{\prime} ; x_{2}\right) & \ldots
\end{array}\right]
$$

is a matrix of size $n_{p} \times n_{c}, n_{c}=2 N_{\max }$, containing complex coefficients derived from the radial basis functions, and

$$
\mathbf{P}=\left[\begin{array}{llll}
\left\{P_{s m 0}^{+} e^{j k_{1}^{+} x_{1}}\right. & \left\{P_{s m 0}^{-} e^{j k_{2}^{-} x_{2}}\right.
\end{array} \quad\left\{P_{s m 1}^{+} e^{j k_{1}^{+} x_{1}}\right\} \quad\left\{P_{s m 1}^{-} e^{j k_{2}^{-} x_{2}}\right\} \quad \cdots\right]^{\mathrm{T}}
$$

is the solution vector of length $n_{c}$ for the complex radial mode amplitudes. By ensuring that the number of measurement points $N_{1}+N_{2}$ is greater than twice the number of radial basis functions $N_{\max }$, the problem is overdetermined, $n_{p}>n_{c}$, and a solution to this linear problem is again easily computed using the singular value decomposition method. More details are given in Dahl et al. ${ }^{2}$

Duct conditions and axial rake locations that would produce a singularity or near singularity in the inverse matrix calculation using $\mathbf{B}$ in equation (6) are to be avoided. Using previous work on errors in two-microphone, in-duct measurements, $\AA \mathrm{bom}^{8}$ provides practical limits that for the no-flow case are given by

$$
0.1 \pi<k_{m n}\left|x_{2}-x_{1}\right|<0.8 \pi
$$


For a hardwall duct, the wave number can be written in terms of the cutoff ratio $\xi$.

$$
k_{m n}=\frac{\omega}{c} \sqrt{1-\frac{1}{\xi^{2}}}
$$

These limits are also followed in the in-duct, two-microphone, higher order mode measurements in Akoum \& Ville. ${ }^{9}$

\section{Rotating Rake Experiments}

The Advanced Noise Control Fan ${ }^{10}$ (ANCF) is a highly configurable 4-foot diameter ducted fan located in the Aero-Acoustic Propulsion Laboratory (AAPL) at the NASA Glenn Research Center. The AAPL is a hemispherical, anechoic (above $125 \mathrm{~Hz}$ ) test facility used for noise measurements. In order to provide a clean, cylindrical duct, for these experiments, the ANCF spool pieces were stacked up in a vertical orientation providing a constant area cylindrical duct. The entire stack rested on the floor with approximately 6 inches of foam material placed in the bottom of the stack to minimize reflections from the floor. A flow lip was attached to the duct exit as shown schematically in Figure 1.

The Configurable Fan Artificial Noise System (CFANS), described by Sutliff, ${ }^{3}$ was utilized to generate and control circumferential modes. The system consists of four axially distributed source rows, each containing 16 electromagnetic drivers distributed around the circumference. There are two spool pieces, each having two source rows of flush mounted drivers on the wall. A computer controlled signal generator was used to send the signals to each driver independently, in the proper relationship, to generate the desired circumferential mode. The signals to each row can be adjusted globally to affect the radial distribution, if desired. The practical limits of the system are circumferential mode orders up to \pm 7 and a maximum frequency of $1500 \mathrm{~Hz}$.

The modes were measured by the Rotating Rake mode measurement system. ${ }^{5}$ Typically, a single rake, extending from the outer-wall to the duct center-line, has been mounted to measure the modes. Since measurements at a single axial location will not be able to account for reflections in the duct, an additional rake was mounted for the experiment on the same rotating ring as the original as shown in the left side of Figure 2. This second rake was adjustable in the axial direction over the range of 2.5 to 10.5 inches, in fixed, one-inch increments, and was mounted 180 degrees in the circumferential direction from the original rake, which remains fixed in the axial direction at a location designated as the origin of the $x$-axis pointing outward from the duct. Figure 2 also shows a close up picture of the separation between the rakes.

Data were obtained from the dual rakes in order to provide the two-point axial variation required to compute the reflection. Reflections were created using two methods. The first method relies on the natural reflections due to an open-ended exit termination. The ring containing the dual rotating rake system was mounted at the open exit of the ANCF duct as shown in Figure 3. The single source row (labelled C) farthest from the exit termination was used to generate the modes that propagate towards the exit. This configuration was used during testing with the flow lip attached and was assumed to minimize reflections. It was also used with the flow lip removed creating a sharp 90 degree flanged exit. This should result in higher levels of reflections compared to when the flow lip was present. A second configuration was used to generate artificial reflections, see Figure 4. This was accomplished by locating the dual rake ring in the center of the duct. Source row C was used to generate the "primary" wave and source row B was used to generate the "reflected" wave. Each source row was actuated independently and the modes were measured by the dual rakes. Then both sets were activated simultaneously and the resulting superposition was measured.

\section{Results with One Source}

Using the configuration shown in Figure 3, the CFANS drivers in source row $\mathrm{C}$ were set to generate a circumferential mode at $m=2$ with a frequency of $480 \mathrm{~Hz}$. Under these conditions only one mode propagates in the ANCF duct, the $(2,0)$ mode. Initially, the flow lip was attached to the duct exit. The lip tends to minimize the reflection of sound within the duct and allows most of the sound generated in the duct to propagate out of the duct. The results for mode amplitudes from one-rake analysis using data from both the fixed rake (Rake 1 shown in Figure 1) and the movable rake (Rake 2) are shown in Figure 5a. The mode amplitudes from the fixed rake data with the lip exit in place are plotted as a function of the movable rake location and give an indication of the repeatability of the measurement. The average fixed rake mode amplitude, $19.24 \pm 0.09$, is plotted at $x=0$. At each of the six movable rake locations, the data for 
the two rakes are collected simultaneously and used to perform the two-rake analysis. The results show a fairly constant mode amplitude in both propagation directions with average values $\left|P_{20}^{+}\right|=18.77 \pm 0.46$ and $\left|P_{20}^{-}\right|=2.82 \pm 0.21$.

The other set of mode amplitude results shown in Figure 5b are for the same sound conditions and set of measurements but with the lip removed from the duct leaving just the mounting flange at the duct exit. It was expected that the sound would more readily reflect back into the duct with this exit condition. The average fixed rake mode amplitude plotted at $x=0$ is $23.84 \pm 0.15$. The results show from the two-rake analysis that, on average, the incident mode amplitude is $\left|P_{20}^{+}\right|=19.66 \pm 0.46$, similar to the incident mode amplitude with the lip in place. However, the average reflected mode amplitude has doubled, $\left|P_{20}^{-}\right|=5.89 \pm 0.29$.

The remaining results in Figure 5 show the computed standing wave amplitudes compared to the one-rake analysis results of the movable rake data for both the lip and the flange conditions at the duct exit. The standing wave amplitude is computed from the equation

$$
\begin{aligned}
A(x) & =\left|P_{m n}^{+} e^{j k x}+P_{m n}^{-} e^{-j k x}\right| \\
& =\left|P_{m n}^{+}\right|\left[1+2|R| \cos \left(2 k x-\theta_{R}+\phi\right)+|R|^{2}\right]^{1 / 2}
\end{aligned}
$$

where $R=P_{m n}^{-} / P_{m n}^{+}$is the complex reflection coefficient at $x=0$ with phase $\theta_{R}$. The phase $\phi$ is used to account for any additional phase shift. The plot of equation (9) with $\phi=0$ for both the lip and flange exit conditions is shown using the broken black lines. The movable rake mode amplitudes determined from one-rake analysis are near the computed standing wave amplitudes. A fit of equation (9), using $\phi$ as the fit parameter, was also performed. This resulted in a shift of the standing wave amplitude curves toward the source as shown by the broken green lines. For the lip exit condition, $\phi=0.22$, and for the flange exit condition, $\phi=0.16$. These translate to distances of $1.57 \mathrm{~cm}$ and $1.08 \mathrm{~cm}$, respectively, for the two exit conditions. These distances represent the combined uncertainties in knowing the locations of the source, the point of reflection, and the measurement microphones.

\section{Open End Reflection Coefficients}

The reflection coefficients for the open end of the duct were determined from measurements using the configuration, shown in Figure 3, with the two rakes near the exit of the duct. Source row $\mathrm{C}$ was used to generate acoustic modes at $480 \mathrm{~Hz}$ and $960 \mathrm{~Hz}$ as listed in Table 1 . The $(4,0)$ mode at $480 \mathrm{~Hz}$ was found to be near the cutoff condition making reliable measurements difficult according to the limits given by equation (8). Hence, no reflection coefficients were determined for this condition. For the remaining seven modes, multiple rake separation measurements were made within the limits specified by equation (8) allowing multiple measurements of the same exit conditions for each mode.

Table 1: Comparison of open end duct reflection coefficient magnitudes from measurements and analytical predictions.

\begin{tabular}{|c|c|c|c|c|c|c|c|}
\hline$f(\mathrm{~Hz})$ & $(m, n)$ & $\xi^{\mathrm{a}}$ & Unflanged $^{\mathrm{b}}$ & $\infty$ Flange $^{\mathrm{c}}$ & Flange $^{\mathrm{d}}$ & Lip $^{\mathrm{d}}$ & Lip $^{\mathrm{e}}$ \\
\hline 480 & 2,0 & 1.743 & $0.240,0.260$ & 0.186 & $0.30 \pm 0.02$ & $0.15 \pm 0.01$ & $0.19 \pm 0.01$ \\
480 & 4,0 & 1.001 & $0.970,0.836$ & 0.953 & - & - & - \\
960 & 2,0 & 3.487 & $0.099,0.116$ & 0.076 & $0.51 \pm 0.04$ & $0.19 \pm 0.05$ & $0.20 \pm 0.03$ \\
960 & 2,1 & 1.588 & $0.088,0.100$ & 0.069 & $0.35 \pm 0.03$ & $0.23 \pm 0.10$ & $0.09 \pm 0.02$ \\
960 & 2,2 & 1.068 & $0.341,0.372$ & 0.291 & $0.33 \pm 0.06$ & $0.05 \pm 0.03$ & $0.05 \pm 0.03$ \\
960 & 4,0 & 2.003 & $0.152,0.171$ & 0.116 & $0.24 \pm 0.08$ & $0.10 \pm 0.03$ & $0.04 \pm 0.02$ \\
960 & 4,1 & 1.147 & $0.223,0.244$ & 0.182 & $0.23 \pm 0.09$ & $0.17 \pm 0.09$ & $0.07 \pm 0.05$ \\
960 & 6,0 & 1.420 & $0.192,0.223$ & 0.152 & $0.23 \pm 0.04$ & $0.11 \pm 0.02$ & $0.13 \pm 0.03$ \\
\hline
\end{tabular}

${ }^{\text {a }}$ cutoff ratio

${ }^{\mathrm{b}}$ Computed from equations in Rienstra ${ }^{11}$ and Lansing, ${ }^{12}$ left number and Cho, ${ }^{13}$ right number.

${ }^{\mathrm{c}}$ Computed from equations in Zorumski ${ }^{14}$ using 10 modes.

$\mathrm{d}$ Two-rake measurements near the exit, Figure 3.

e Two-rake measurements mid duct, Figure 4. 
Measured reflection coefficients with the no-lip or flange exit condition are compared to predicted unflanged and infinite flanged duct reflection coefficients in Table 1. Rienstra ${ }^{11}$ and Lansing et al. ${ }^{12}$ derived analytical solutions for an unflanged duct with negligibly thin walls. Computed results for their formulations were identical. $\mathrm{Cho}^{13}$ applied statistical theory to the same configuration. Reflection coefficients were similar in magnitude to the analytical approaches. These results are shown separately in the table. The infinite flange reflection coefficients were computed using the analytical equations given by Zorumski. ${ }^{14}$ These are all less than the unflanged duct reflection coefficients. This is in agreement with expectations inferred from the results shown in $\mathrm{Cho}^{15}$ for the limits on the general condition of a hyperboloid shape at the duct exit. The physical duct in this experiment has a finite width flange for which there is no complete analytic theory for arbitrary incident modes. Ando ${ }^{16}$ has completed an analysis for an incident plane wave. Those results indicate that the magnitude of the reflection coefficient increases from the unflanged case as the exit flange or duct wall width increases. From the analysis and results shown in Ando ${ }^{16}$ and in Cho, ${ }^{15}$ it is inferred that the reflection coefficient for an open end duct with a finite flange should be between the limiting values for the reflection coefficients of the unflanged and the infinite flanged ducts. The measured reflection coefficients for the flanged duct shown in Table 1 are closer to the analytical unflanged duct predictions. The largest discrepancies are for the $(2,0)$ and $(2,1)$ modes at $960 \mathrm{~Hz}$.

The differences between measured reflection coefficients and predictions can be high. Ville \& Silcox ${ }^{17}$ have differences from $25 \%$ to $100 \%$ between measured and predicted reflection coefficients using modes $(2,0),(4,0)$, and $(6,0)$ in an unflanged duct. Typically higher differences were found closer to mode cutoff with decreasing differences as the frequency increased. Their measurements were made with microphones mounted in the duct wall at multiple axial locations. Akoum \& Ville ${ }^{9}$ found similar levels of differences for measurements in a flanged duct compared to predictions. In this case, a two-microphone method was used with the axially separated pair traversed around inside of the duct. The amount of data presented here is insufficient to be any more accurate than these previous experimental measurements. However, the discrepancies for the $(2,0)$ and $(2,1)$ modes at $960 \mathrm{~Hz}$ appear excessive compared to what was presented in Ville \& Silcox ${ }^{17}$ and in Akoum \& Ville ${ }^{9}$ and warrant further investigation.

Table 1 also shows the measured reflection coefficient magnitudes for the duct with the lip exit condition using the rake configuration in Figure 3. Though not clearly shown, the inner surface of the lip angles away from the duct axis at about 60 degrees. From the results in Cho, ${ }^{15}$ it is inferred that the magnitude of the reflection coefficient for an infinite 60 degree hyperboloid surface should be less than that of the infinite flange reflection coefficient, which is less than the unflanged case. The measured reflection coefficients with the lip in place are less, as expected, than the measured reflection coefficients for the flange condition for all modes. Ville \& Silcox ${ }^{17}$ found similar reductions in the magnitudes of the reflection coefficients for all modes measured when the end of the unflanged duct was replaced with a 57 degree bellmouth. Similar low reflection coefficients for the lip exit condition were also measured during testing with the rake configuration shown in Figure 4.

\section{Results with Two Sources}

Tests were conducted with a variety of frequencies, mode numbers, and source levels using the configuration shown in Figure 4. The data from the two rotating rakes were used to compute the mode amplitudes. First, as a single rake analysis for both the fixed rake and the movable rake. Then, as a dual rake analysis to determine the mode amplitudes for the $+x$ direction propagating mode, which is the direction outward from the duct, and the $-x$ direction propagating mode. A first set of example results is shown in Figure 6 where the frequency is nominally $480 \mathrm{~Hz}$ and the $(2,0)$ mode propagates. The amplitude gain $B_{g}$ for the sources in row $\mathrm{B}$ was varied over $1.0,0.75,0.5$, and 0.25 . The figure shows mode amplitudes for $B_{g}=0.75$ in the left column and $B_{g}=0.25$ in the right column. The top row results are for both the $\mathrm{B}$ and $\mathrm{C}$ source rows operating. The middle row results are for source row B only and the bottom row is for source row $\mathrm{C}$ only. Since the row $\mathrm{C}$ sources were set the same for both the measurements with $B_{g}=0.25$ and the measurements with $B_{g}=0.75$, the results in Figures $6 \mathrm{e}$ and $6 \mathrm{f}$ are basically the same and give an indication of measurement repeatability. The changes in $B_{g}$ are directly reflected in the mode amplitude results shown in Figures $6 \mathrm{c}$ and $6 \mathrm{~d}$. The $-x$ direction mode amplitude decreases as $B_{g}$ decreases and the level of any reflection off the treatment surface also decreases as seen in the level of the $+x$ direction mode amplitudes. With both source rows $\mathrm{B}$ and $\mathrm{C}$ operating, the $-x$ direction mode amplitudes again decrease as $B_{g}$ decreases while the $+x$ direction mode amplitudes are about constant since they are controlled by the constant source row C levels. 
The standing wave pattern measured by the movable rake is much larger when both source levels are nearly the same as shown in Figure 6a compared to the much less prominent standing wave results in Figure 6b when the source row B levels are low.

A second set of example mode amplitude results is shown in Figure 7 where the nominal frequency is $960 \mathrm{~Hz}$ and the $(4,1)$ mode propagates in the duct. These results show the same behavior as the B source gain changes as seen in Figure 6.

The measured data from the two rotating rakes between two source rows was used to determine the $+x$ direction and the $-x$ direction propagating mode amplitudes. In general, the $+x$ direction mode amplitudes were relatively constant as the source levels at row $\mathrm{C}$ were held constant. In contrast, the $-x$ direction mode amplitudes varied as the amplitude level gain of source row B varied. To show this, the $-x$ direction mode amplitude as the gain $B_{g}$ changes was normalized by the $-x$ direction mode amplitude determined when $B_{g}=1$. The results are plotted in Figure 8. The results for various modes when only source row B operates is shown in black. Ideally, as the source gain changes, the mode amplitude changes by the same amount. The black symbols tend to cluster near the ideal line given that there is some scatter in the results and that the treatment is not a perfect absorber. The red symbols are the same normalized mode amplitude results for the $-x$ direction modes when sound was generated from both source rows B and C. The normalizing $-x$ direction mode amplitude with $B_{g}=1$ was determined with source row $\mathrm{C}$ operating. These results deviate more from the ideal results.

To better explain the measured results, a simple model was developed using the method of images. Given the position of the microphones in the fixed rake between the two source rows B and C was designated as the origin, $x=0$, of the $x$-axis system pointing outward from the duct, the source row $\mathrm{B}$ is at the location $x=L_{B}$ and source row $\mathrm{C}$ is at $x=-L_{C}$. In the region $-L_{C} \leq x \leq L_{B}$, the sound from source row $\mathrm{C}$ and from an image source of $\mathrm{C}$ on the opposite side of a reflection point at the duct exit, $x=L_{E}$, is given by

$$
P_{1}(x)=P_{C} e^{j k\left(x+L_{C}\right)}+R_{E} P_{C} e^{-j k\left(x-2 L_{E}-L_{C}\right)}
$$

A similar equation for source row B and its image source opposite the treated surface at $x=-L_{W}$ is given by

$$
P_{2}(x)=P_{B} e^{-j k\left(x-L_{B}\right)}+R_{W} P_{B} e^{j k\left(x+2 L_{W}+L_{B}\right)}
$$

The complex factors $R_{E}$ and $R_{W}$ are reflection coefficients at the exit of the duct and at the treated surface at the closed end of the duct, respectively. Using superposition

$$
P(x)=P_{1}(x)+P_{2}(x)=P^{+} e^{j k x}+P^{-} e^{-j k x}
$$

where

$$
\begin{aligned}
& P^{+}=P_{C} e^{j k L_{C}}+R_{W} P_{B} e^{j k\left(2 L_{W}+L_{B}\right)}=P_{C} e^{j k L_{C}}+\bar{R}_{W} P_{B} e^{j k L_{B}} \\
& P^{-}=P_{B} e^{j k L_{B}}+R_{E} P_{C} e^{j k\left(2 L_{E}+L_{C}\right)}=P_{B} e^{j k L_{B}}+\bar{R}_{E} P_{C} e^{j k L_{C}}
\end{aligned}
$$

The right side of equation (13) follows from the single source measurements of the reflection coefficients at $x=0$. Using only source row $\mathrm{B}$, the reflection coefficient for the wall treatment translated back to $x=0$ is $\bar{R}_{W}=R_{W} \exp \left(j k 2 L_{W}\right)$. Similarly using only source row $\mathrm{C}$, the reflection coefficient for the open end is $\bar{R}_{E}=R_{E} \exp \left(j k 2 L_{E}\right)$. These equations model the wave in the $+x$ direction as being the sum of a wave directly generated by source row $\mathrm{C}$ plus the wave generated by source row $\mathrm{B}$ that reflects off the treated wall and travels back up the duct in the $+x$ direction. Similarly, the wave traveling in the $-x$ direction consists of the wave directly from source row B plus that portion of the wave from source row $\mathrm{C}$ that reflects at the exit and returns down the duct in the $-x$ direction. The model can be made more complicated by adding more image sources. For instance, an image source can be added for the wave reflected by the treated wall from source row C. Also, this analysis neglects the effects of the source impedance, which Munjal \& Eriksson ${ }^{18}$ consider to be inappropriate, and assumes the source region to not reflect, but allow transmission of waves not initially generated by the source. However, when normalized as is done below, equations derived based on the work of Munjal \& Eriksson, including source impedance, reduce to a similar set of equations.

We now take $P^{+}$and $P^{-}$and multiply by their conjugate to get

$$
\left|P^{+}\right|^{2}=\left|P_{C}\right|^{2}+2\left|\bar{R}_{W}\right|\left|P_{B}\right|\left|P_{C}\right| \cos \left[k\left(L_{B}-L_{C}\right)+\theta_{R W}+\theta_{B}-\theta_{C}\right]+\left|\bar{R}_{W}\right|^{2}\left|P_{B}\right|^{2}
$$

and

$$
\left|P^{-}\right|^{2}=\left|P_{B}\right|^{2}+2\left|\bar{R}_{E}\right|\left|P_{C}\right|\left|P_{B}\right| \cos \left[k\left(L_{C}-L_{B}\right)+\theta_{R E}+\theta_{C}-\theta_{B}\right]+\left|\bar{R}_{E}\right|^{2}\left|P_{C}\right|^{2}
$$


where the $\theta$ 's are the phases of their associated complex values. Normalizing equations (14) and (15) by the value of $\left|P^{-}\right|$determined when the $\mathrm{C}$ source is set to zero and the amplitude gain $B_{g}=1$, that is $\left|P_{B 0}^{-}\right|$, we get equations that are normalized mode power

$$
\begin{gathered}
\frac{\left|P^{+}\right|^{2}}{\left|P_{B 0}^{-}\right|^{2}}=B_{g}^{2}\left|\bar{R}_{W}\right|^{2} C_{0}^{2}+B_{g} 2 C_{1} C_{0}\left|\bar{R}_{W}\right| \cos \left[k\left(L_{B}-L_{C}\right)+\theta_{R W}+\phi\right]+C_{1}^{2} \\
\frac{\left|P^{-}\right|^{2}}{\left|P_{B 0}^{-}\right|^{2}}=B_{g}^{2} C_{0}^{2}+B_{g} 2 C_{1} C_{0}\left|\bar{R}_{E}\right| \cos \left[k\left(L_{C}-L_{B}\right)+\theta_{R E}-\phi\right]+\left|\bar{R}_{E}\right|^{2} C_{1}^{2}
\end{gathered}
$$

where

$$
\frac{\left|P_{B}\right|}{\left|P_{B 0}^{-}\right|} \propto B_{g}=C_{0} B_{g}
$$

indicates that the source row B levels decrease as the gain setting is reduced, $C_{1}$ is a constant

$$
C_{1}=\frac{\left|P_{C}\right|}{\left|P_{B 0}^{-}\right|}
$$

since the source row $\mathrm{C}$ level is held constant and $\phi$ represents an unknown phase shift due to a phase difference between sources B and $\mathrm{C}$ and any distance uncertainties. Results from measured data for different gain settings $B_{g}$ were used in a least-squares fit to equations (16) and (17) to determine the fit parameters $C_{0}, C_{1}$ and $\phi$. The results of the fit are given in Table 2 for the seven modes. The values for the magnitude of the reflection coefficient $R_{W}$ for the treated wall were determined from measurements using only source row $\mathrm{B}$ and are included in the table. The reflection coefficient magnitudes for $R_{E}$ are shown in the last column of Table 1.

Table 2: Parameters determined from fitting equations (16) and (17) to results from measured data.

\begin{tabular}{|c|c|c|c|c|c|}
\hline$f(\mathrm{~Hz})$ & $(m, n)$ & $C_{0}$ & $C_{1}$ & $\phi$ & $\left|R_{W}\right|$ \\
\hline 480 & 2,0 & 1.023 & 1.277 & 1.116 & 0.22 \\
960 & 2,0 & 1.177 & 1.046 & 0.072 & 0.24 \\
960 & 2,1 & 0.920 & 1.025 & 0.358 & 0.29 \\
960 & 2,2 & 1.177 & 1.169 & -1.571 & 0.19 \\
960 & 4,0 & 1.014 & 0.797 & 1.264 & 0.32 \\
960 & 4,1 & 1.106 & 1.238 & 0.655 & 0.07 \\
960 & 6,0 & 0.957 & 1.263 & 1.342 & 0.12 \\
\hline
\end{tabular}

The curve fit results for equation (17), the normalized mode power for the $P^{-}$mode amplitude, are plotted in Figure 9a for different frequencies and propagating mode numbers. Noting the low values for $\left|R_{E}\right|$ in Table 1 , we see that very little energy reflects back from the duct exit for as $B_{g}$ gets small and source row $\mathrm{C}$ remains constant, the power in the $P^{-}$wave decreases proportionally to the decrease in the source row B power. Figure $9 \mathrm{~b}$ shows the curve fit results for equation (16), the normalized mode power for the $P^{+}$ mode amplitude. Even though $P^{+}$may be dominated by the wave generated from source row $\mathrm{C}$, which is held constant, only one, the $(4,1)$ mode at $960 \mathrm{~Hz}$, has constant power with changes in $B_{g}$ since that mode has the lowest treated wall reflection coefficient. For the other frequencies and mode numbers, the sloping line indicates interference between the $P^{+}$wave generated by source row $\mathrm{C}$ and the treated wall reflected wave from source row B. A positive slope with increasing $B_{g}$ shows constructive interference. Conversely, a negative slope with increasing $B_{g}$ indicates destructive interference.

\section{Concluding Remarks}

The rotating rake mode measurement data analysis process was extended from the analysis of data from one rake to include the data simultaneously collected from a co-rotating second rake. Since measurements at one rake location do not provide sufficient data to determine the direction of propagating modes, a second 
rake measurement axially separated from the first rake is minimally necessary. The analysis of the data from each rake is the same, providing total circumferential mode amplitudes at each microphone location. By taking the data from two microphone rakes simultaneously, changes with axial distance are captured in the total circumferential mode amplitudes. The two sets of total circumferential mode amplitudes are considered to be the sum of radial basis functions half of which are for modes propagating in one direction and the other half propagating in the other direction. Hence, using a least-squares fit to the total circumferential mode amplitudes from both locations, the mode amplitudes that apply to modes propagating in both directions are obtained. The method ensures that evanescent modes are properly included in the fit.

Experiments were conducted using artificial acoustic sources generating modal content in the duct to be measured by the rotating rakes. Using a single source, standing waves were generated between the source and the open end of the duct. A single rake measured the mode amplitude of a standing wave and with measurements from two rakes, the standing wave was decomposed into the modes propagating in each direction. This data were used to determine the reflection coefficients for the open end of the duct. Compared to analytical predictions, discrepancies with measured reflection coefficients could be large. Other studies using different measurement methodologies had similar levels of discrepancy. Results from rake measurements between two sources showed that the method could distinguish the behavior of the propagating modes in each direction as one source varied in amplitude.

\section{Acknowledgment}

The NASA Fixed Wing Project of the Fundamental Aeronautics Program supported this work.

\section{References}

\footnotetext{
${ }^{1}$ Sutliff, D. L., "Turbofan Duct Mode Measurements Using a Continuously Rotating Microphone Rake," Int. J. of Aeroacoustics, Vol. 6, No. 2, 2007, pp. 147-170.

${ }^{2}$ Dahl, M. D., Hixon, R., and Sutliff, D. L., "Further Development of Rotating Rake Mode Measurement Data Analysis," AIAA Paper No. 2013-2246, 2013.

${ }^{3}$ Sutliff, D. L., "A Mode Propagation Database Suitable for Code Validation Utilizing the NASA Glenn Advanced Noise Control Fan and Artificial Sources," AIAA Paper No. 2014-0719, 2014.

${ }^{4}$ Dahl, M. D. and Sutliff, D. L., "Numerical Technique for Analyzing Rotating Rake Mode Measurements in a Duct With Passive Treatment and Shear Flow," AIAA Paper No. 2007-3679, 2007.

${ }^{5}$ Sutliff, D. L., "Rotating Rake Turbofan Duct Mode Measurement System," NASA TM-2005-213828, October 2005.

${ }^{6}$ Lawson, C. L. and Hanson, R. J., Solving Least Squares Problems, Prentice-Hall, Englewood Cliffs, NJ, 1974.

${ }^{7}$ Ovenden, N. C. and Rienstra, S. W., "Mode-Matching Strategies in Slowly Varying Engine Ducts," AIAA J., Vol. 42, No. 9, September 2004, pp. 1832-1840.

8 Åbom, M., "Modal Decomposition in Ducts Based on Transfer Function Measurements Between Microphone Pairs," J. Sound Vib., Vol. 135, No. 1, 1989, pp. 95-114.

${ }^{9}$ Akoum, M. and Ville, J.-M., "Measurement of the Reflection Matrix of a Discontinuity in a Duct," J. Acoust. Soc. Am., Vol. 103, 1998, pp. 2463-2468.

${ }^{10}$ Loew, R. A., Lauer, J. T., McAllister, J., and Sutliff, D. L., "The Advanced Noise Control Fan," AIAA Paper No. 2006-3150, 2006

${ }^{11}$ Rienstra, S. W., "Acoustic Radiation from a Semi-Infinite Annular Duct in a Uniform Subsonic Mean Flow," J. Sound Vib., Vol. 94, No. 2, 1984, pp. 267-288.

${ }^{12}$ Lansing, D. L., Drischler, J. A., and Pusey, C. G., "Radiation of Sound from an Unflanged Circular Duct with Flow," 79th Meeting of the Acoustical Society of America, 1970.

${ }^{13}$ Cho, Y. C., "A Statistical Theory for Sound Radiation and Reflection from a Duct," J. Acoust. Soc. Am., Vol. 65, No. 6, 1979, pp. 1373-1379.

${ }^{14}$ Zorumski, W. E., "Generalized Radiation Impedances and Reflection Coefficients of Circular and Annular Ducts," J. Acoust. Soc. Am., Vol. 54, No. 6, 1973, pp. 1667-1673.

${ }^{15}$ Cho, Y. C., "Rigorous Solutions for Sound Radiation from Circular Ducts with Hyperbolic Horns or Infinite Plane Baffle," J. Sound Vib., Vol. 69, No. 3, 1980, pp. 405-425.

${ }^{16}$ Ando, Y., "On the Sound Radiation from Semi-Infinite Circular Pipe of Certain Wall Thickness," Acustica, Vol. 22, No. 4, 1969, pp. 219-225.

${ }^{17}$ Ville, J.-M. and Silcox, R. J., "Experimental Investigation of the Radiation of Sound From an Unflanged Duct and a Bellmouth, Including the Flow Effect," NASA TP-1697, 1980.

${ }^{18}$ Munjal, M. L. and Eriksson, L. J., "Analysis of a Linear One-Dimensional Active Noise Control System by Means of Block Diagrams and Transfer Functions," J. Sound Vib., Vol. 129, No. 3, 1989, pp. 443-455.
} 


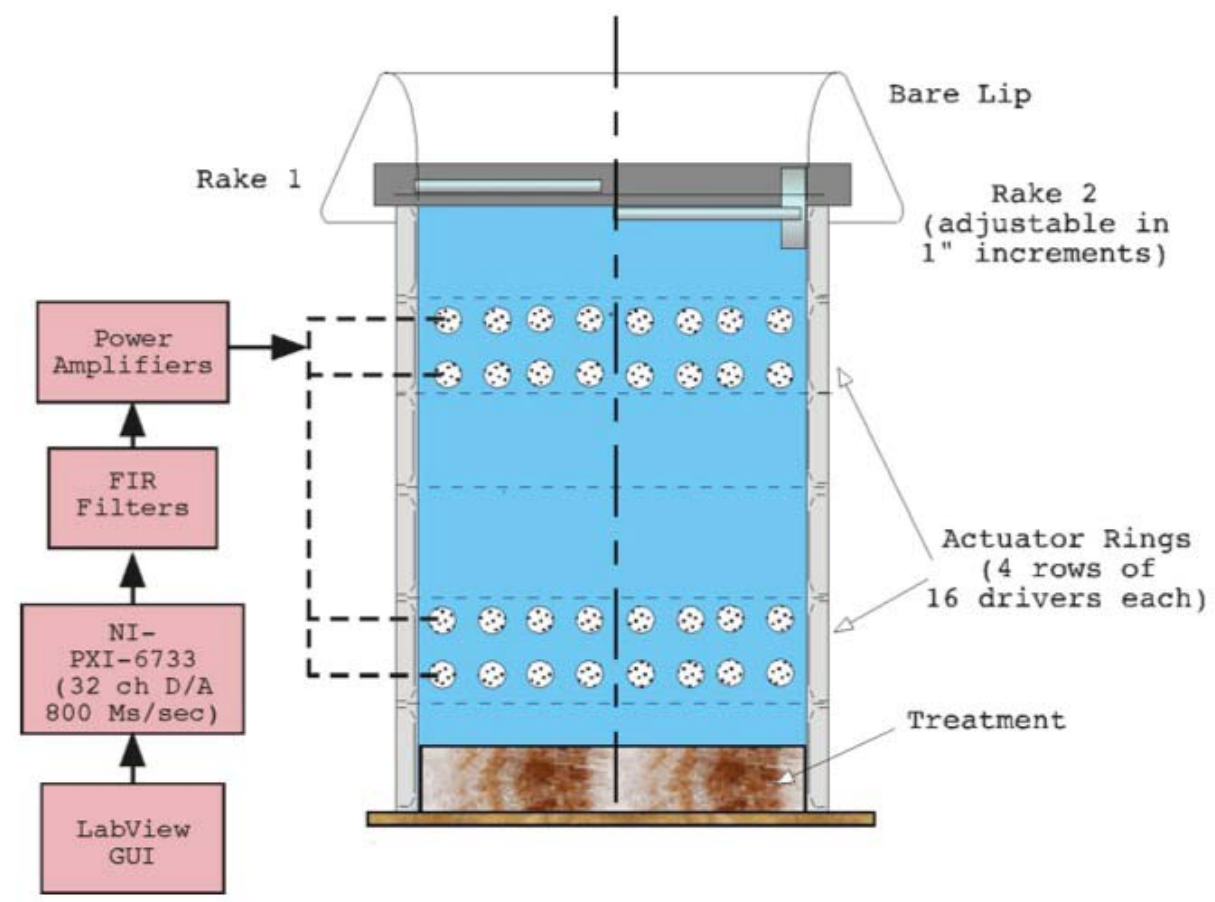

Figure 1: Schematic diagram of the Advanced Noise Control Fan duct with Configurable Fan Artificial Noise System and rotating rakes installed.
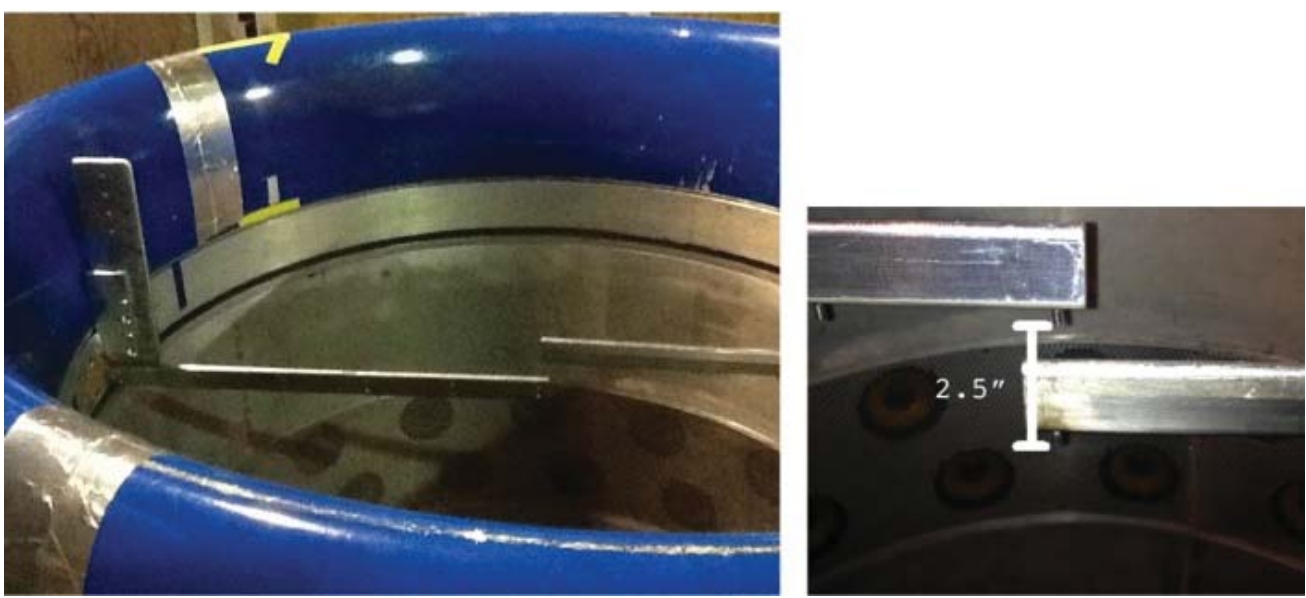

Figure 2: Pictures of the two rotating rakes installed near the lip of the duct and a close-up of the rakes with a 2.5 inch separation distance. 


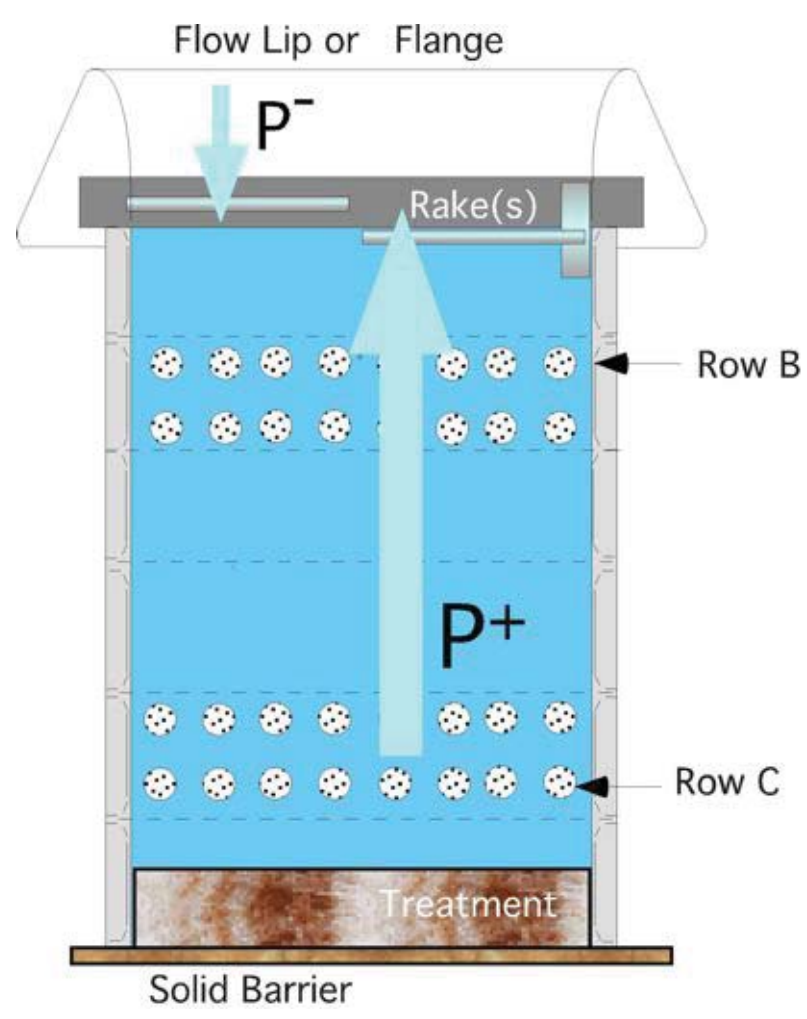

Figure 3: ANCF in mode reflection configuration where the reflection from the duct termination is measured.

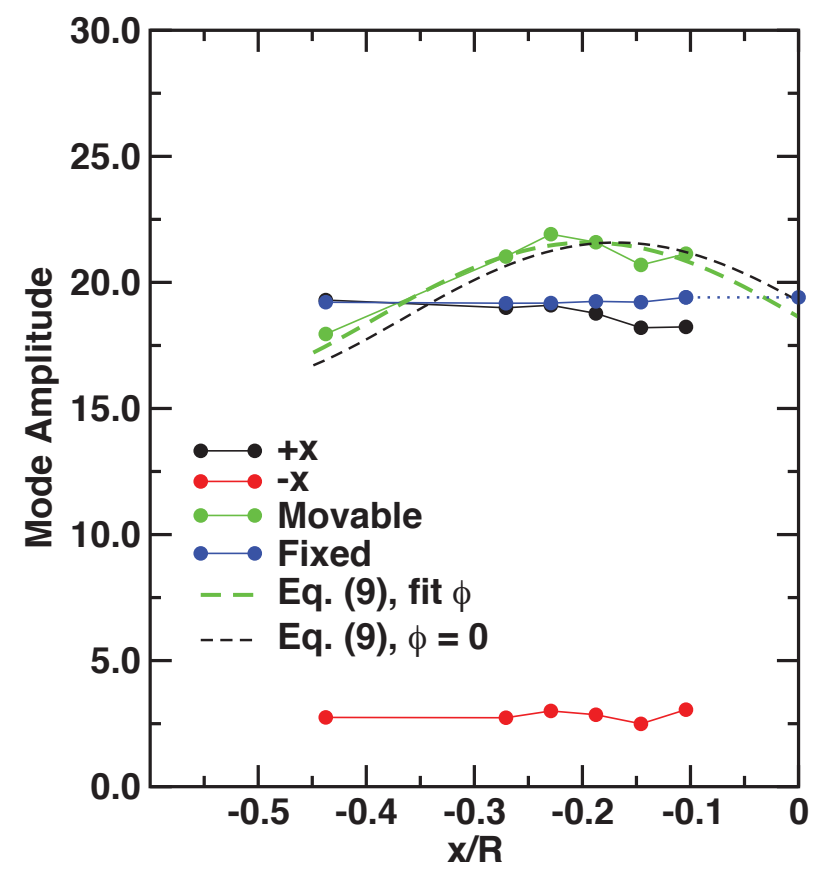

(a) Lip mounted at exit

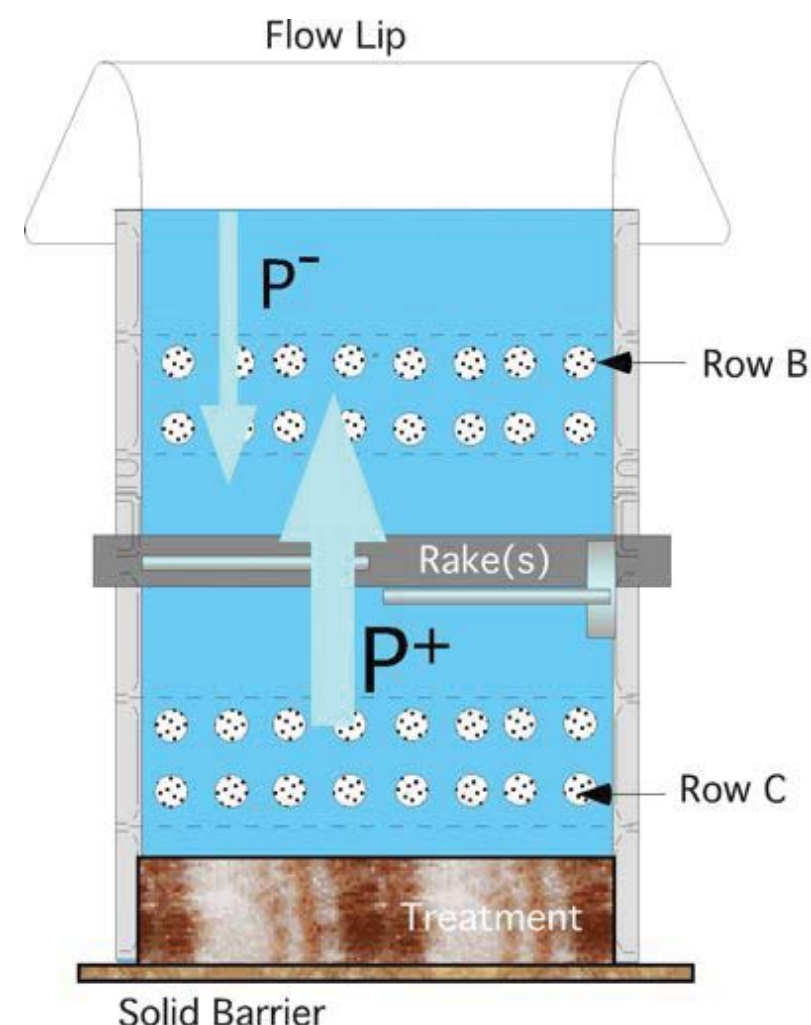

Figure 4: ANCF in mode reflection configuration where an artificial reflection is generated and the modes are measured between the two sets of sources.

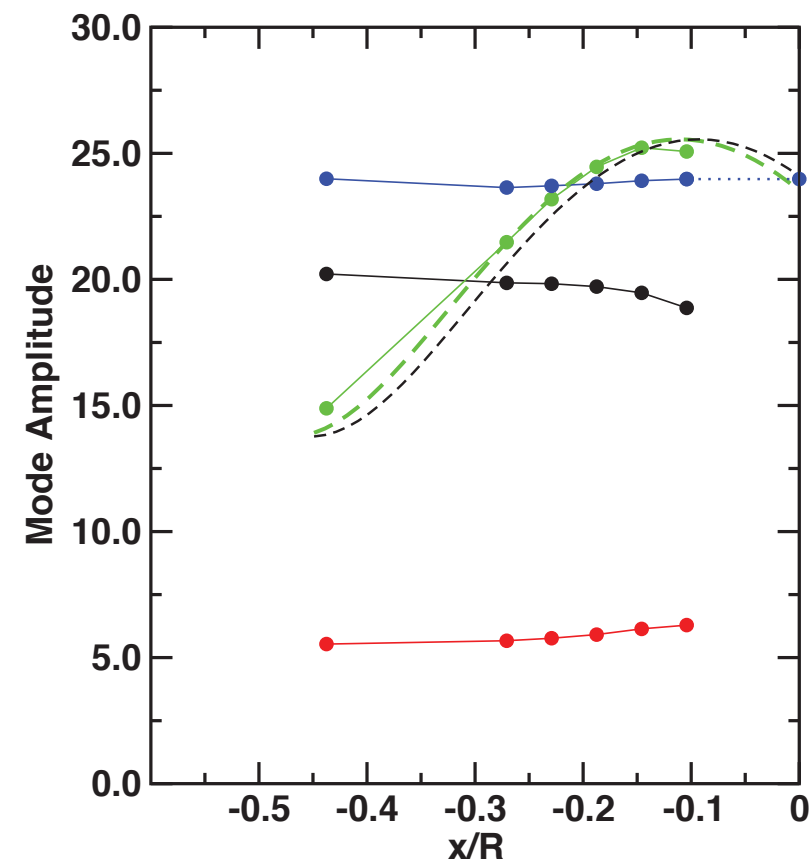

(b) No-lip or flange exit

Figure 5: Comparison of one-rake and two-rake analyses for mode amplitude using ANCF measured data with different exit conditions. $480 \mathrm{~Hz}$, mode $(2,0)$. Source row C. 


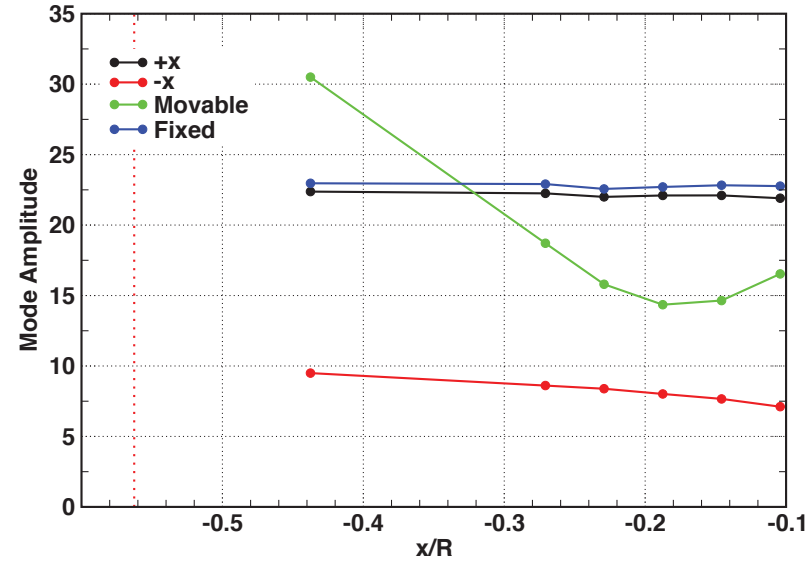

(a) Source rows B and C, $B_{g}=0.75$

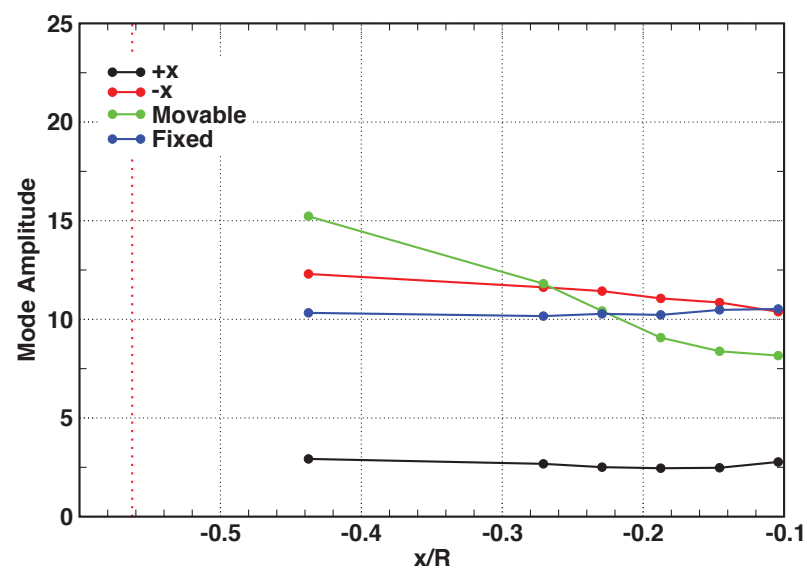

(c) Source row B, $B_{g}=0.75$

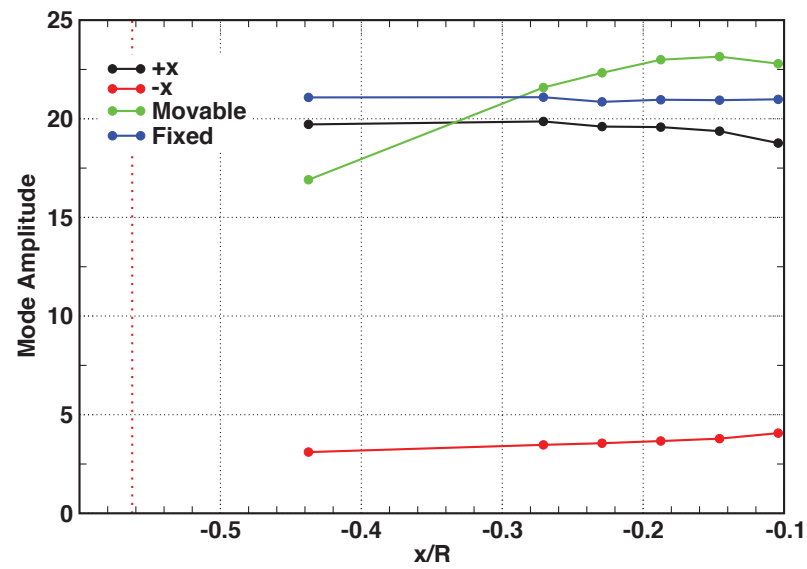

(e) Source row C

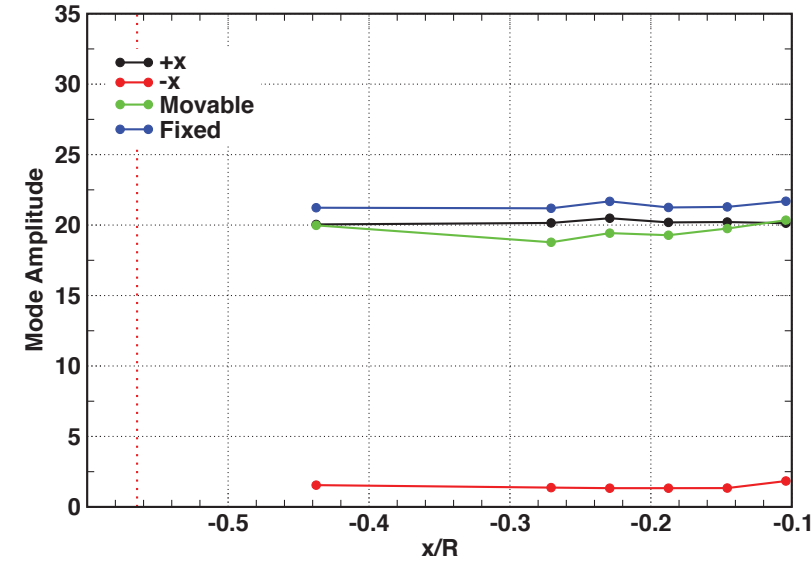

(b) Source rows B and C, $B_{g}=0.25$

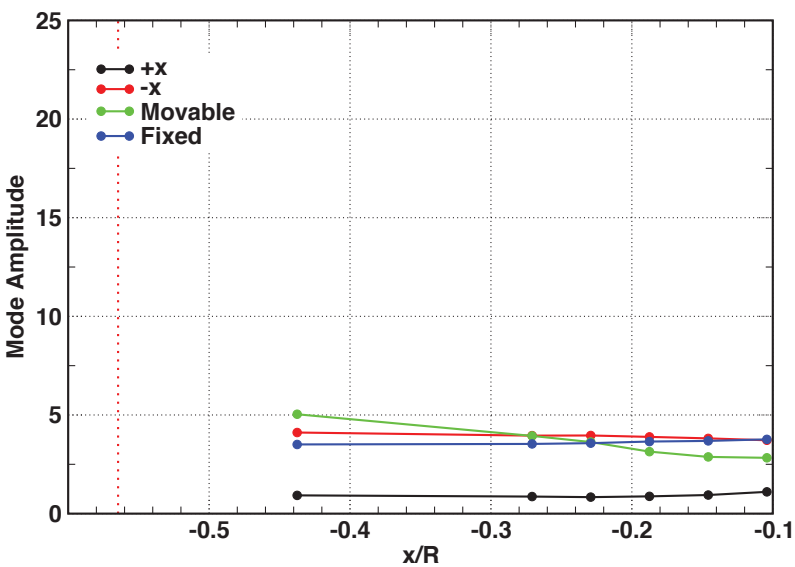

(d) Source row B, $B_{g}=0.25$

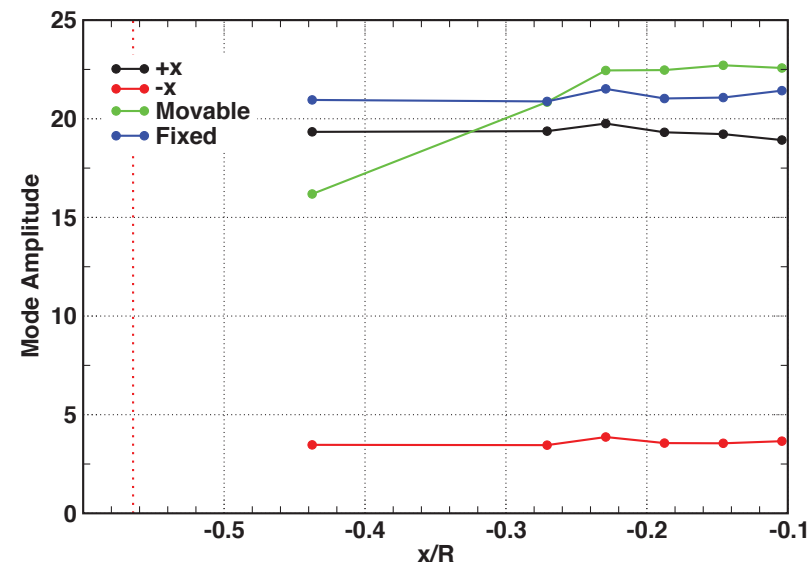

(f) Source row C

Figure 6: Comparison of one-rake and two-rake analyses for mode amplitude using ANCF measured data with two sources (Figure 4). $480 \mathrm{~Hz}$, mode $(2,0)$. Red dash line shows upper limit of eq. (8). 


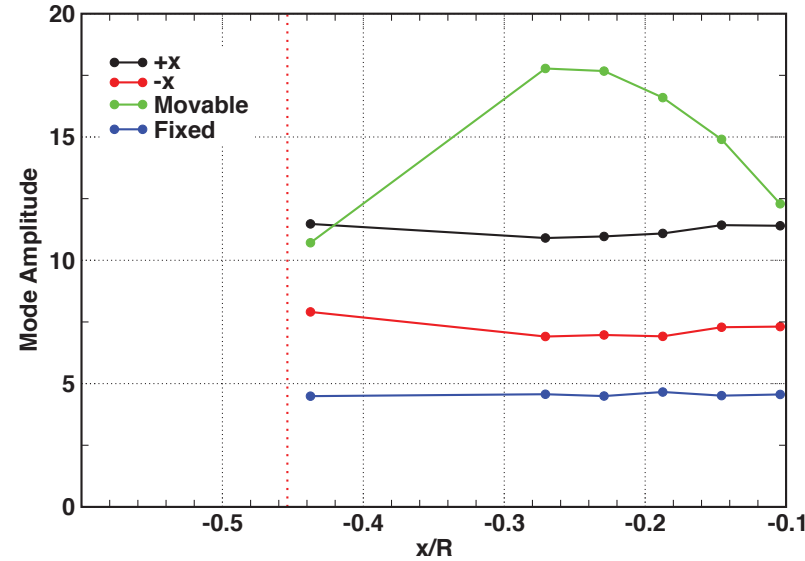

(a) Source rows B and C, $B_{g}=0.75$

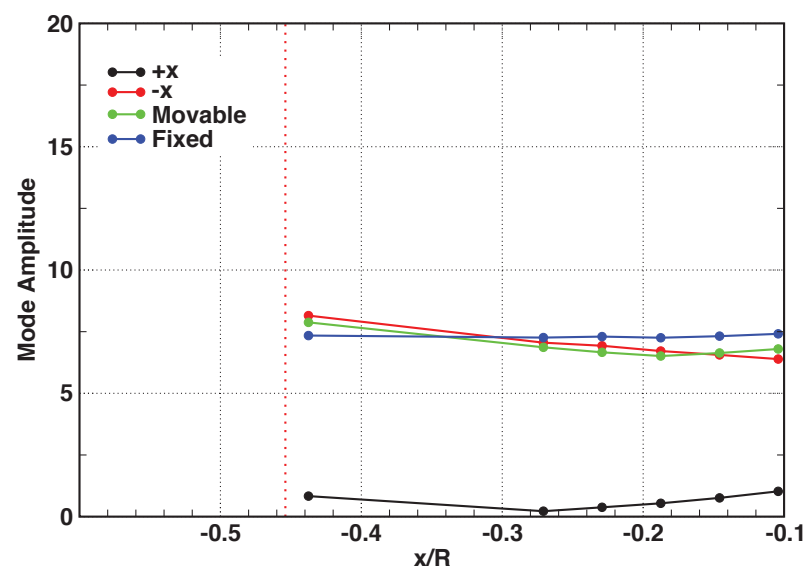

(c) Source row B, $B_{g}=0.75$

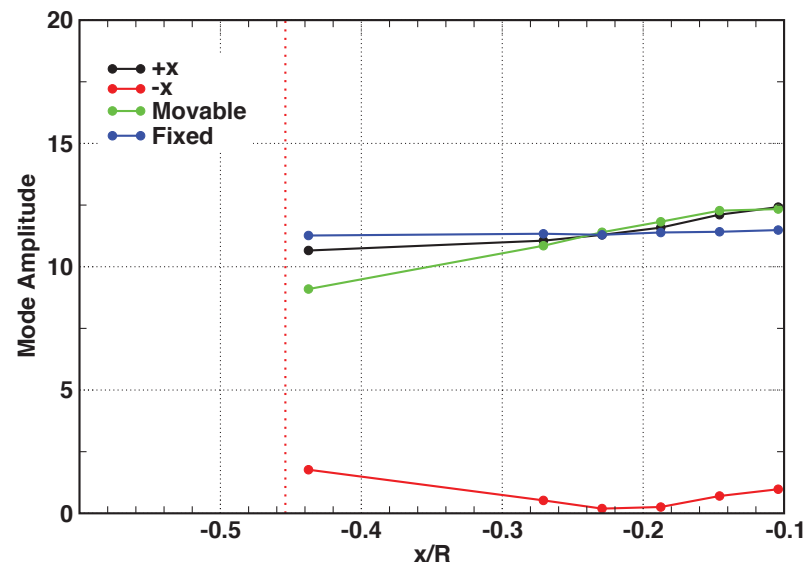

(e) Source row C

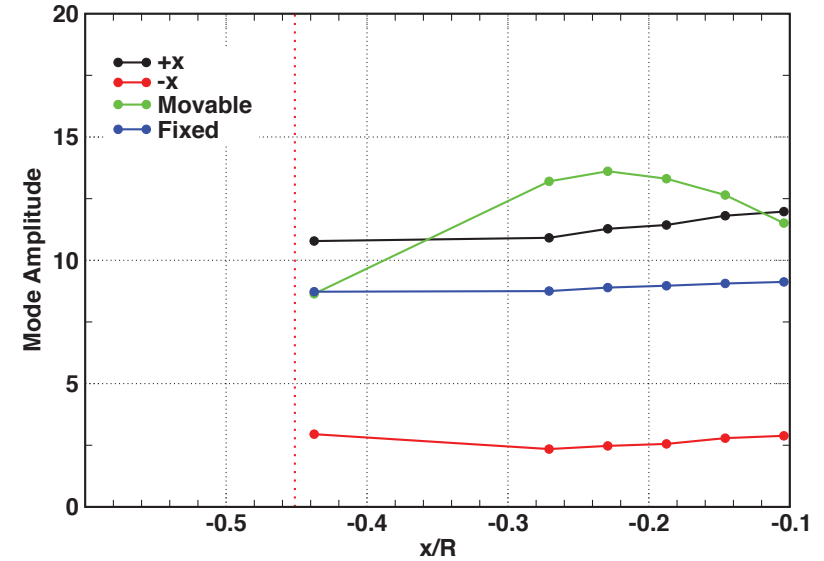

(b) Source rows B and C, $B_{g}=0.25$

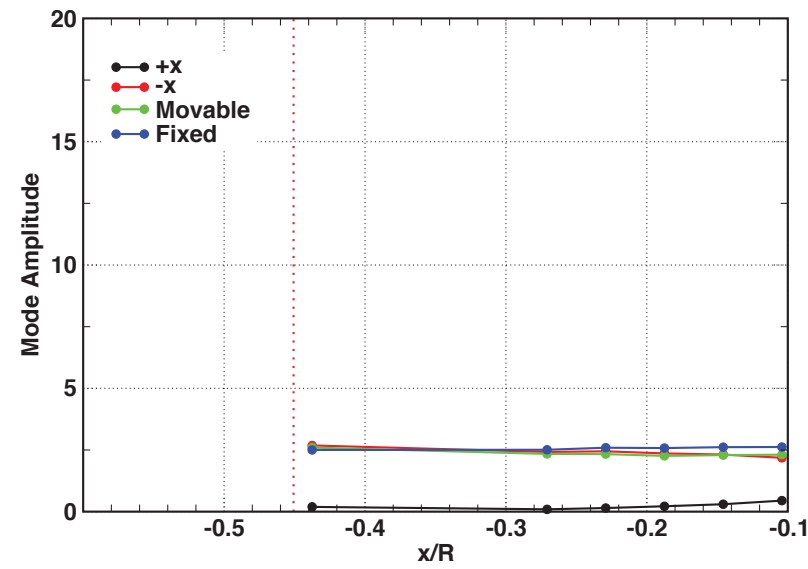

(d) Source row B, $B_{g}=0.25$

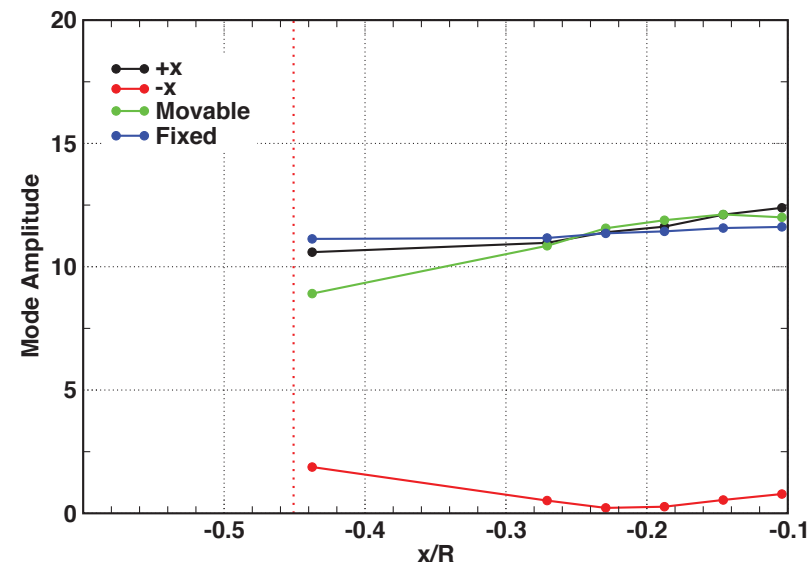

(f) Source row C

Figure 7: Comparison of one-rake and two-rake analyses for mode amplitude using ANCF measured data with two sources (Figure 4). $960 \mathrm{~Hz}$, mode $(4,1)$. Red dash line shows upper limit of eq. (8). 


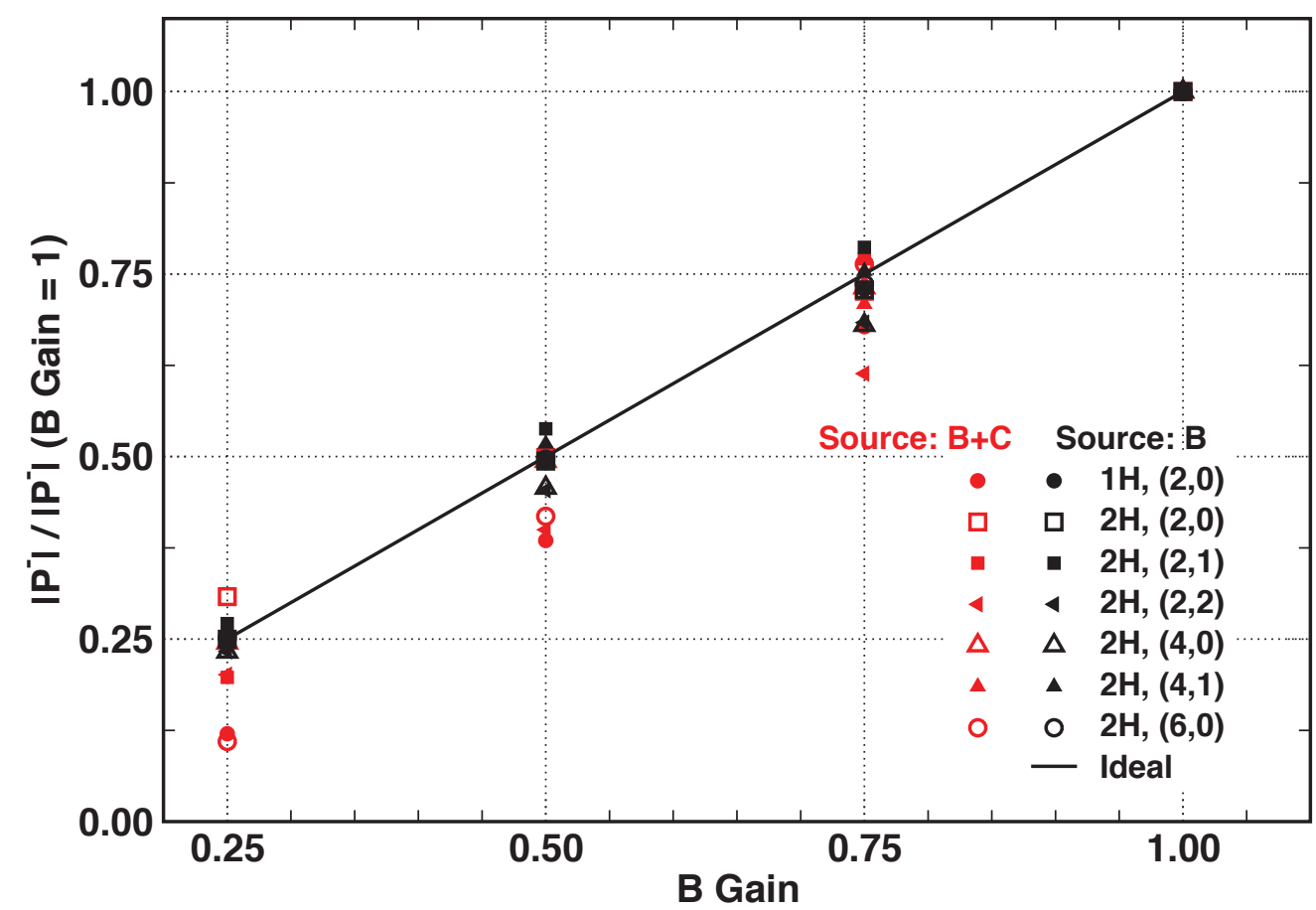

Figure 8: Relative change of the $-x$ direction propagating modes $P^{-}$as measured in the configuration of Figure 4 with either source row B alone or source rows B and C. $1 \mathrm{H}$ is $480 \mathrm{~Hz} .2 \mathrm{H}$ is $960 \mathrm{~Hz}$.

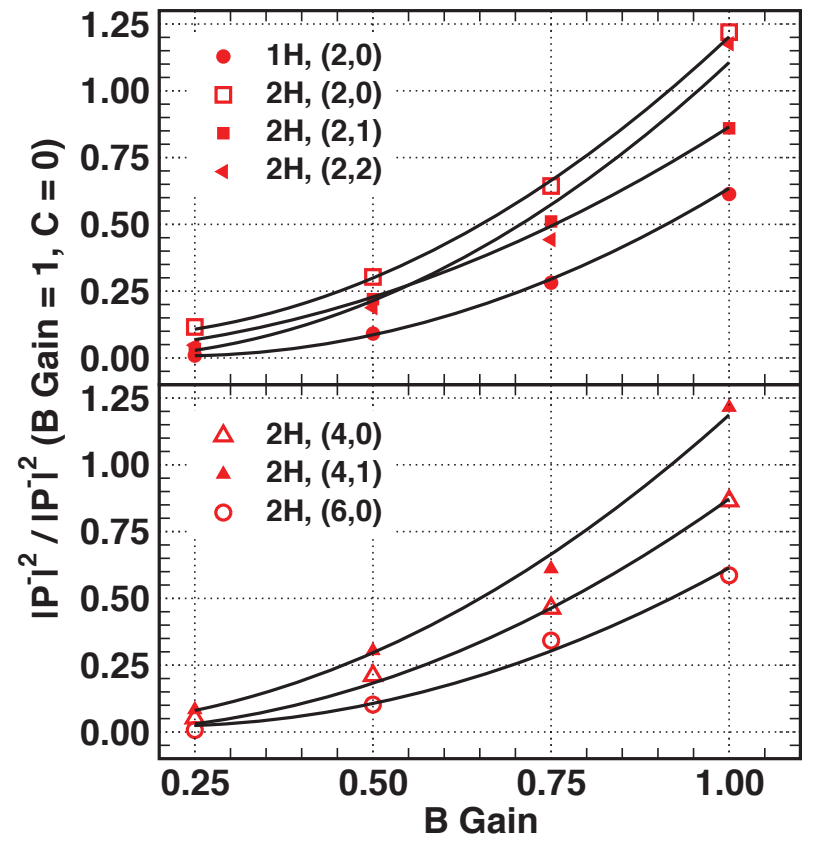

(a) Normalized $P^{-}$mode power and eq. (17)

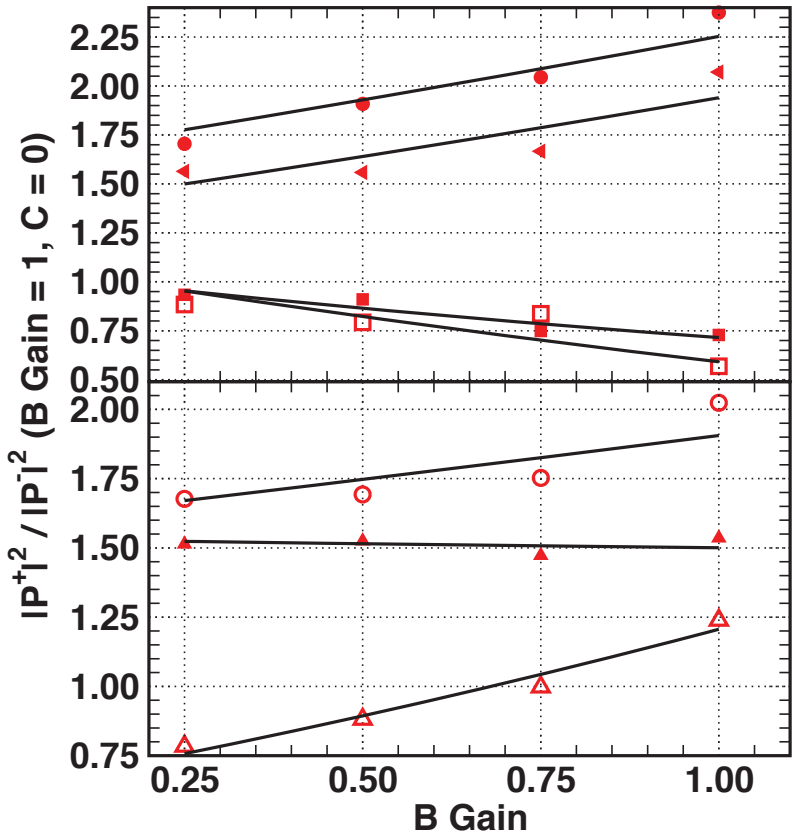

(b) Normalized $\mathrm{P}^{+}$mode power and eq. (16)

Figure 9: Relative change of the directional propagating modes as measured in the configuration of Figure 4 with source rows B and C. Lines are the curve fits of the data. $1 \mathrm{H}$ is $480 \mathrm{~Hz}$. $2 \mathrm{H}$ is $960 \mathrm{~Hz}$. 